\title{
Embrittlement of Metal by Solute Segregation-Induced Amorphization
}

\author{
Hsiu-Pin Chen, ${ }^{1}$ Rajiv K. Kalia, ${ }^{1}$ Efthimios Kaxiras,${ }^{2}$ Gang Lu, ${ }^{3}$ Aiichiro Nakano, ${ }^{1}$ Ken-ichi Nomura, ${ }^{1}$ \\ Adri C. T. van Duin, ${ }^{4}$ Priya Vashishta, ${ }^{1}$ and Zaoshi Yuan ${ }^{1}$ \\ ${ }^{1}$ Collaboratory for Advanced Computing and Simulations, Department of Physics \& Astronomy, \\ Department of Chemical Engineering \& Materials Science, Department of Computer Science, University of Southern California, \\ Los Angeles, California 90089-0242, USA \\ ${ }^{2}$ Department of Physics, Harvard University, Cambridge, Massachusetts 02138, USA \\ ${ }^{3}$ Department of Physic and Astronomy, California State University Northridge, Northridge, California 91330-8268, USA \\ ${ }^{4}$ Department of Mechanical and Nuclear Engineering, Pennsylvania State University, University Park, Pennsylvania 16802, USA
} (Received 25 November 2009; published 16 April 2010)

\begin{abstract}
Impurities segregated to grain boundaries of a material essentially alter its fracture behavior. A prime example is sulfur segregation-induced embrittlement of nickel, where an observed relation between sulfur-induced amorphization of grain boundaries and embrittlement remains unexplained. Here, $48 \times$ $10^{6}$-atom reactive-force-field molecular dynamics simulations provide the missing link. Namely, an orderof-magnitude reduction of grain-boundary shear strength due to amorphization, combined with tensilestrength reduction, allows the crack tip to always find an easy propagation path.
\end{abstract}

DOI: 10.1103/PhysRevLett.104.155502

PACS numbers: $62.20 . \mathrm{mm}, 81.05 . \mathrm{Bx}, 81.07 . \mathrm{Bc}$

The modification of chemical bonds due to a small amount of impurities segregated to grain boundaries (GBs) controls the mechanical properties of materials [1]. Despite decades of intense experimental [2,3] and theoretical [4-6] efforts, however, mechanisms of such GB mechanochemistry [7] are not well understood. In the case of sulfur (S) segregation-induced embrittlement of nickel $(\mathrm{Ni})$, which is important for the development of next-generation nuclear reactors [8], Heuer et al. performed tensile tests of notched specimens with varying amount of $\mathrm{S}$ segregation to GBs [3]. (The maximum range of $\mathrm{S}$ segregation was determined to be $0.5 \mathrm{~nm}$ on either side of a GB.) They observed a transition from transgranular ductile fracture to intergranular brittle fracture at a critical S concentration of $15.5 \pm 3.4 \%$ at GBs. In addition, they measured another critical $\mathrm{S}$ concentration for amorphization of Ni face-centered cubic (fcc) crystal during $\mathrm{S}^{+}$-ion implantation [3]. The critical S concentration of $14.2 \pm$ $3.3 \%$ for amorphization coincides with the critical S concentration for GB embrittlement within experimental error. These experiments clearly demonstrate an essential relation between amorphization and embrittlement. The central question is: What are the atomistic mechanisms that relate amorphization to embrittlement?

Answering this question poses a so called multiscale simulation challenge [9], i.e., coupling quantummechanical accuracy to describe solute chemistry with large length scales to incorporate long-range stress fields and microstructures such as grains and amorphous GB phases. Recent developments in chemically reactive atomistic simulation methods and parallel computing technologies that are scalable over $10^{5}$ processors (see [10], Fig. S1) [11], combined with those in nanomechanical experiments [12], have set the stage to address this challenge. Namely, simulations and experiments can now study the mechanical properties of nanoscale materials at the same length scale. Here, we perform large molecular dynamics (MD) simulations (see [10], Supplementary Methods) based on reactive force fields (REAXFF) [13,14], which are trained and validated (Supplementary Tables S1 and S2, [10]) by quantum-mechanical calculations based on the density functional theory [15], in order to study the effect of S segregation in nanocrystalline $\mathrm{Ni}$.

We first investigate the effect of S doping on the structure of Ni crystal. Here, we perform REAXFF MD simulation of fcc $\mathrm{Ni}$, where a fraction of $\mathrm{Ni}$ atoms in the range of $0 \%-24 \%$ is randomly substituted by $\mathrm{S}$ atoms. The system is a cube of side $3.52 \mathrm{~nm}$ with periodic boundary conditions. For each $\mathrm{S}$ concentration, the system is relaxed by a steepest-descent procedure and is further relaxed for $5 \mathrm{ps}$ at $10 \mathrm{~K}$, before it is gradually heated up to $300 \mathrm{~K}$. Figure 1(a) compares the radial distribution functions, $g(r)$, for $0 \%$ and $24 \%$ S substitutions. We observe broadening of the peaks in the latter, which represents the disordering of the fcc structure due to S doping. The FWHM of the first peak of $g(r)$ as a function of S concentration in Fig. 1(b) exhibits a sudden increase at $16 \%$, which is close to the experimental threshold $(14.2 \pm 3.3 \%)$ for amorphization [3]. In fact, Fig. 1(c) shows that the bond angle distribution at $24 \% \mathrm{~S}$ substitution closely resembles that of amorphous Ni prepared by a melt-quench procedure. The amorphization threshold, 16\%, is close to the percolation threshold [16], $14 \%$, for randomly substituted $\mathrm{S}$ in fcc $\mathrm{Ni}$, considering the connectivity up to the second nearest-neighbor $\mathrm{S}$ atoms at $0.35 \mathrm{~nm}$. In the light of strong S-S interaction in Ni up to $0.33 \mathrm{~nm}$ as pointed out in Ref. [4], it is reasonable to identify the amorphization as a percolation transition with $0.35 \mathrm{~nm}$ connectivity. In contrast, the $16 \%$ amorph- 

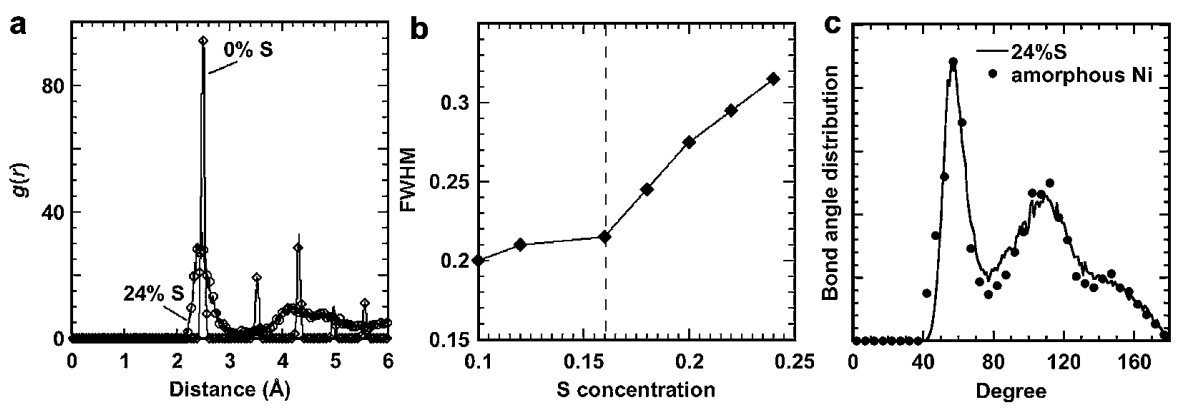

FIG. 1. S-induced amorphization of Ni crystal. (a) Radial distribution function $g(r)$ of Ni fcc crystal with $0 \%$ and $24 \% \mathrm{~S}$ doping. (b) Full width at half maximum (FWHM) of the first peak of $g(r)$ as a function of S concentration. (c) Bond angle distribution of Ni fcc crystal with $24 \% \mathrm{~S}$ doping and that of amorphous Ni.

ization threshold is away from the percolation threshold, $20 \%$, considering only the nearest-neighbor S-S $(0.25 \mathrm{~nm})$ or $7 \%$ for the third nearest-neighbor S-S $(0.5 \mathrm{~nm})$. The latter may indicate the disappearance of S-S interaction at $0.5 \mathrm{~nm}$, which may explain the experimentally observed amorphous GB sulfide thickness of $0.5 \mathrm{~nm}$.

To study the effect of S-induced GB amorphization on the fracture behavior of $\mathrm{Ni}$, we perform REAXFF MD simulations for two systems: Pure Ni nanocrystal (NC-Ni) and Ni nanocrystal with $20 \%$ S-doped GB layers of thickness $0.5 \mathrm{~nm}(\mathrm{NC}-\mathrm{Ni}+20 \% \mathrm{~S})$ [17]. A notch is inserted to each sample [Fig. 2(a)], and fracture simulations are performed by applying uniaxial strain perpendicular to the crack plane until the system fragments into two separate pieces. Each nanocrystalline $\mathrm{Ni}$ sample consists of 48393282 atoms (a total of 2048 grains), with dimensions $468.0 \mathrm{~nm} \times$ $234.1 \mathrm{~nm} \times 4.9 \mathrm{~nm}(\mathrm{NC}-\mathrm{Ni})$ or $470.4 \mathrm{~nm} \times 235.2 \mathrm{~nm} \times$ $4.9 \mathrm{~nm}(\mathrm{NC}-\mathrm{Ni}+20 \% \mathrm{~S})$ in the $x, y$ and $z$ directions. The two nanocrystalline configurations consisting of randomly oriented grains of average size $7.5 \mathrm{~nm}$ are generated using the Voronoi tessellation method [18] with the same grain geometry. Samples with several different grain geometries have been made to provide statistics. We first relax the atomic configurations and the simulation box size with periodic boundary conditions over 50 ps until the pressure reaches zero, followed by further relaxation for $50 \mathrm{ps}$ at $10 \mathrm{~K}$ and subsequent heating up to $300 \mathrm{~K}$. A notch of length $150 \mathrm{~nm}$ is inserted in the $x z$ plane of each sample, which is then fully relaxed again by removing the periodic boundary condition in the $x$ direction [Fig. 2(a)]. Fracture simulations are performed by applying uniaxial strain along the $y$ direction at a strain rate of $4 \times 10^{8} \mathrm{~s}^{-1}$. The tensile strain is applied by changing the simulation box length in the $y$ direction, while periodic boundary conditions are maintained in both $y$ and $z$ directions. The computation has taken 10 days on 65536 IBM BlueGene/P processors at Argonne National Laboratory.

Movies of these simulations exhibit distinct fracture behavior: i.e., ductile tearing in NC-Ni (Supplementary Movie S1, [10]) and brittle cleavage (Supplementary Movie S2, [10]) in $\mathrm{NC}-\mathrm{Ni}+20 \% \mathrm{~S}$. Figure 2 shows a crossover from mixed transgranular and intergranular fracture in NC-Ni [Fig. 2(b)] to purely intergranular fracture in $\mathrm{NC}-\mathrm{Ni}+20 \% \mathrm{~S}$ [Fig. 2(c)]. In NC-Ni, the crack propagates through both grains [transgranular fracture indicated by white arrows in Fig. 2(b)] and GBs (intergranular fracture), resulting in $56 \%$ intergranular fracture. Common ductile fracture behaviors such as crack-tip blunting, void formation in front of the crack tip and its coalescence with the main crack, and necking are observed in $\mathrm{NC}-\mathrm{Ni}$, in agreement with experimental observations [19]. Figure 2(b) also shows a void extending several GBs, which may explain dimple microstructures observed in fractured NC-Ni surfaces [19]. In contrast, the crack in NC-Ni $+20 \% \mathrm{~S}$ propagates in a cleavage manner only through GBs, resulting in $100 \%$ intergranular fracture, in agreement with experiments [3].

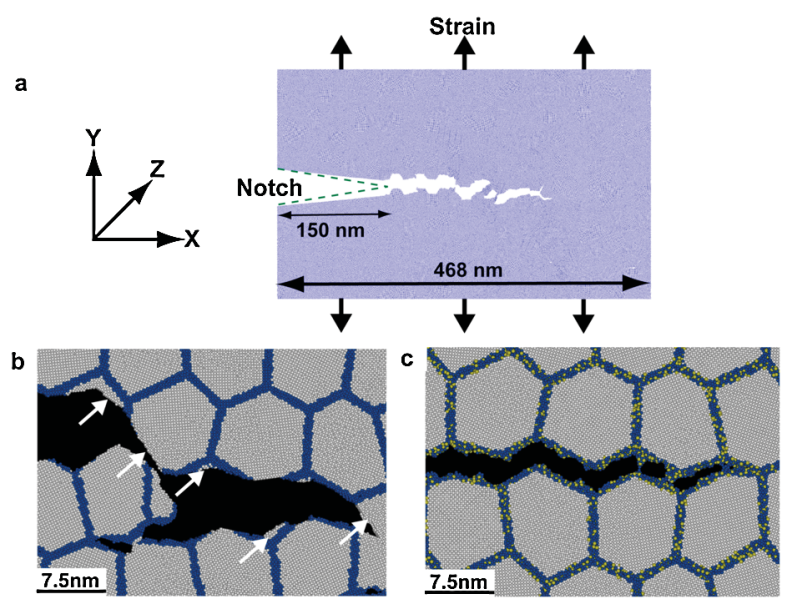

FIG. 2 (color). Crossover of fracture modes caused by amorphous sulfide intergranular phases in nanocrystalline Ni. (a) A snapshot of MD simulation, where prenotched nanocrystalline $\mathrm{Ni}$ is fractured by applying uniaxial strain. (b) and (c) are closeups of the fracture simulations for $\mathrm{NC}-\mathrm{Ni}$ and $\mathrm{NC}-\mathrm{Ni}+20 \% \mathrm{~S}$, respectively, where gray, blue and yellow colors represent $\mathrm{Ni}$ atoms inside grains $(>0.5 \mathrm{~nm}$ from GBs), Ni atoms within $0.5 \mathrm{~nm}$ from GBs, and $\mathrm{S}$ atoms, respectively. White arrows in (b) point to transgranular fracture surfaces. 
To determine the mechanism of S-induced transitions of fracture behavior, we first adopt a theoretical framework by Rice and Wang [6], which explains GB embrittlement from the energetics of solute segregation. We perform a tensile test, where the energy required to separate a Ni $\Sigma 5(012)$ symmetric tilt GB is computed with and without a monolayer of segregated $\mathrm{S}$ atoms using REAXFF (see [10]). The calculated work of interfacial separation is reduced from $1.65 \mathrm{~J} / \mathrm{m}^{2}$ for pure Ni GB to $1.20 \mathrm{~J} / \mathrm{m}^{2}$ with segregated $\mathrm{S}$. From the derivative of the energy-separation relation with respect to separation, we also estimate the tensile stress required to separate the GB. The results show the reduction of tensile (cohesive) strength from 22.0 GPa to $15.5 \mathrm{GPa}$ due to $\mathrm{S}$ segregation (Fig. S2, [10]). This suggests S-induced reduction of GB tensile strength as part of the embrittlement mechanism in agreement with Yamaguchi et al. [4]. However, this mechanism alone cannot explain the role of amorphization for embrittlement.

A clue for additional embrittlement mechanism lies in a generalized stacking fault energy (GSFE) [20], i.e., the energy required to slide Ni $\Sigma 5(012) \mathrm{GB}$, without S segregation [Fig. 3(a)], with a monolayer of segregated $\mathrm{S}$ atoms [Fig. 3(b)], and with $0.5 \mathrm{~nm}$-thick amorphous sulfide $\left(\mathrm{Ni}_{0.8} \mathrm{~S}_{0.2}\right)$ GB layers [Fig. 3(c)]. The GSFE is calculated by displacing the upper grain with respect to the lower grain, while atoms are allowed to relax only in the vertical direction [Fig. 3(g)]. In Figs. 3(d)-3(f), shear stress is calculated from the derivative of the three GSFEs [Figs. 3(a)$3(c)]$ with respect to displacement along the [100] direction. The GB shear strength (i.e., the maximum shear

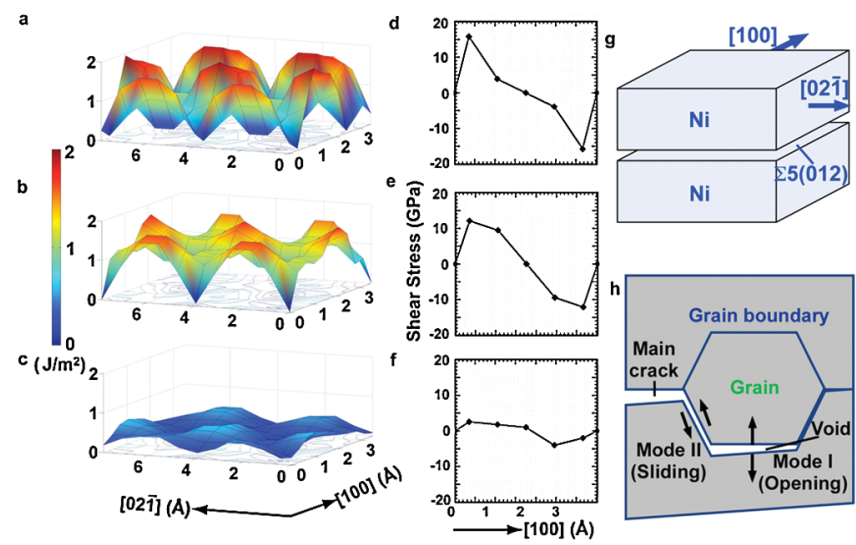

FIG. 3 (color). Grain-boundary shear-strength reduction by S-induced amorphization. Generalized stacking fault energy (GSFE) of Ni $\Sigma 5(012)$ GB without S (a), with a monolayer of segregated S (b), and with amorphous sulfide GB phase (c). Also shown are shear stress calculated from the derivative of the GSFE with respect to displacement along the [100] direction without $S(d)$, with a monolayer of segregated $S(e)$, and with amorphous sulfide GB phase (f). (g) Schematic showing the sliding of one grain with respect to the other to calculate GSFE. (h) Schematic of opening (mode-I) and sliding (mode-II) fractures in front of the main crack. stress) drastically decreases from $17.3 \mathrm{GPa}$ [without $\mathrm{S}$, in Fig. 3(d)] to $2.5 \mathrm{GPa}$ [with amorphous sulfide GB phase, in Fig. 3(f)], due to GB amorphization. In contrast, the $S$ monolayer does not decrease the GB shear strength much [Fig. 3(e)] [21]. This amorphization-induced GB shearstrength reduction provides a hitherto missing link between GB amorphization and embrittlement. As Fig. 3(h) illustrates, the shear-strength reduction provides sliding (mode II) fracture paths in front of the crack tip if the GB ahead of the crack tip is not perpendicular to the tensile direction, while the S-induced tensile-strength reduction provides crack-opening (mode I) paths as in the Rice-Wang model $[4,6]$. Together, the two mechanisms always provide weak cleavage paths right in front of the crack tip, obviating the void opening that is a signature of ductile fracture in pure Ni [Fig. 2(b)], and making the fracture with S segregation purely intergranular and brittle. Sliding of unaccommodated GBs has been postulated as a failure mechanism of NC-Ni [19], which is made easy here by the soft amorphous intergranular phases. Such GB phases endow broad materials with unique mechanical properties, e.g., superhard nanocrystalline ceramics [22].

Because of the reduction of GB shear strength by amorphization, plastic activity within grains [23] is suppressed. We use a common neighborhood parameter (CNP) [24] to identify topological defects such as dislocations and twins during the fracture simulations in $\mathrm{NC}-\mathrm{Ni}$ and $\mathrm{NC}-\mathrm{Ni}+20 \% \mathrm{~S}$. In $\mathrm{NC}-\mathrm{Ni}$, dislocations formed at a GB (dislocation source) are absorbed by another GB (dislocation sink). Figure 4(a) is a snapshot (color-coded by CNP) of ductile fracture in $\mathrm{NC}-\mathrm{Ni}$, where the green stripes show grains that have deformation twinning. The atomic configuration of the twinning structure in Fig. 4(b) is similar to those observed experimentally [25], and such localized plasticity in NC-Ni is consistent with experimental observations [19]. These plastic activities inside grains are largely suppressed in $\mathrm{NC}-\mathrm{Ni}+20 \% \mathrm{~S}$, where plastic deformations are confined in soft amorphous GB phases because GBs fail under shear before dislocations within grains nucleate. The number of intragrain defect atoms (as identified by the CNP) is plotted as a function of time in Fig. 4(c) to provide evidence of the amorphous layer confined plasticity. Here, the large number of defects in NC-Ni represents massive intragrain slip activities, where the ascending and descending parts of the curve correspond to the development of dislocations and their absorption at GBs, respectively. In contrast, limited plasticity inside the grains for $\mathrm{NC}-\mathrm{Ni}+20 \% \mathrm{~S}$ is reflected in the small number of defects in Fig. 4(c).

Another signature of embrittlement is the reduction of toughness, which is a consequence of reduced plastic activity. Figure 4(d) compares the stress-strain relations calculated ahead of the notch for NC-Ni and NC-Ni + $20 \% \mathrm{~S}$. The toughness (the area under the stress-strain curve) is $0.101 \mathrm{GJ} / \mathrm{m}^{3}$ and $0.0898 \mathrm{GJ} / \mathrm{m}^{3}$ for $\mathrm{NC}-\mathrm{Ni}$ 


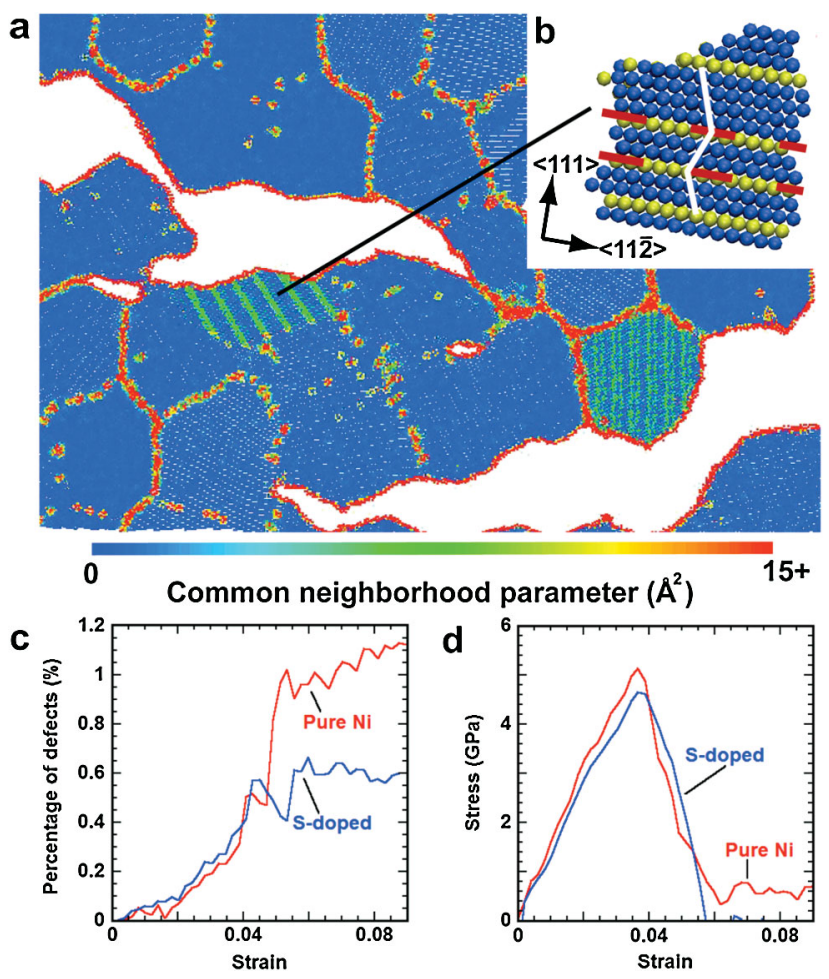

FIG. 4 (color). Ductility of nanocrystalline Ni with and without $\mathrm{S}$ segregation. (a) Close-up of fractured $\mathrm{NC}-\mathrm{Ni}$, where the atoms are color-coded according to the common neighborhood parameter (blue represents atoms with perfect fcc structure). Green stripes represents twinning boundaries. (b) The inset magnifies a deformation twinning in one of the grains, where yellow and blue colors denote the twinning boundary and perfect atoms, respectively. (c) Number of intragrain defect atoms for $\mathrm{NC}-\mathrm{Ni}$ (red) and NC-Ni $+20 \% \mathrm{~S}$ (blue). (d) Stress-strain relation ahead of the notch for NC-Ni (red) and NC-Ni $+20 \% \mathrm{~S}$ (blue).

and NC-Ni $+20 \% \mathrm{~S}$, respectively. Furthermore, the stressstrain curve for $\mathrm{NC}-\mathrm{Ni}+20 \% \mathrm{~S}$ exhibits the characteristics of brittle fracture, i.e., a sudden drop of the stress to zero at a critical strain. In contrast, the stress-strain curve of NC$\mathrm{Ni}$ exhibits nonzero stress up to much larger strains-a characteristic ductile behavior [26].

This work was supported by the Scientific Discovery through Advanced Computing Program of the U.S. Department of Energy, DOE-SciDAC Grant No. DEFC02-06ER25788. The MD simulations were performed on the IBM BlueGene/P computer at Argonne National Laboratory. We thank Professor Ted Lee for discussions.

[1] R. P. Messmer and C. L. Briant, Acta Metall. Mater. 30, 457 (1982).

[2] H. C. Rogers, Science 159, 1057 (1968).

[3] J. K. Heuer et al., J. Nucl. Mater. 301, 129 (2002).

[4] M. Yamaguchi, M. Shiga, and H. Kaburaki, Science 307, 393 (2005).

[5] R. Schweinfest, A. T. Paxton, and M. W. Finnis, Nature (London) 432, 1008 (2004).

[6] J. R. Rice and J. S. Wang, Mater. Sci. Eng. 107, 23 (1989).

[7] J. J. Gilman, Science 274, 65 (1996).

[8] T. R. Allen et al., Nuclear Technology 162, 342 (2008).

[9] S. Yip, Handbook of Materials Modeling (Springer, Berlin, 2005).

[10] See supplementary material at http://link.aps.org/ supplemental/10.1103/PhysRevLett.104.155502.

[11] A. Nakano et al., Int. J. High Perform. Comput. Appl. 22, 113 (2008).

[12] J. R. Greer et al., Adv. Funct. Mater. 19, 2880 (2009).

[13] K. Nomura et al., Comput. Phys. Commun. 178, 73 (2008).

[14] K. D. Nielson et al., J. Phys. Chem. A 109, 493 (2005).

[15] P. Hohenberg and W. Kohn, Phys. Rev. 136, B864 (1964).

[16] D. Stauffer and A. Aharony, Introduction To Percolation Theory (Taylor \& Francis, London, UK, 1994).

[17] As expected, with $20 \%$ S doping, GB sulfide layers have amorphized during sample preparation.

[18] A. Hasnaoui, H. Van Swygenhoven, and P. M. Derlet, Science 300, 1550 (2003).

[19] K. S. Kumar, H. Van Swygenhoven, and S. Suresh, Acta Mater. 51, 5743 (2003).

[20] G. Lu et al., Phys. Rev. B 62, 3099 (2000).

[21] Quantum-mechanical calculation based on density functional theory confirms that a monolayer of S at GB does not reduce the shear strength.

[22] I. Szlufarska, A. Nakano, and P. Vashishta, Science 309, 911 (2005).

[23] J. Li et al., Nature (London) 418, 307 (2002).

[24] H. Tsuzuki, P. S. Branicio, and J. P. Rino, Comput. Phys. Commun. 177, 518 (2007).

[25] C. B. Thomson and V. Randle, Scr. Mater. 35, 385 (1996).

[26] The plasticity of nanocrystalline $\mathrm{Ni}$ is known to be reduced compared with that of $\mathrm{Ni}$ single crystal, see D. Farkas, S. van Petegem, P.M. Derlet, and H. van Swygenhoven, Acta Mater. 53, 3115 (2005). 